\title{
LA DIFUSIÓN E IMPLEMENTACIÓN DE INICIATIVAS DE ORDENACIÓN ESPACIAL SUPRANACIONAL EN EUROPA: APRECIACIÓN DE ALGUNOS EJEMPLOS DE NIVEL INTERMEDIO
}

\author{
Sergio CARAMELO \\ Universidad de Salamanca
}

\section{INTRODUCCIÓN.}

Los procesos de globalización y de integración económica europea no han estado exentos de una rápida mutabilidad territorial y de un enorme impacto socio-económico. Una de las manifestaciones más tangibles de esta realidad la encontramos en el mantenimiento/incremento de fuertes disparidades espaciales y en el desarrollo de conjuntos espaciales y sistemas productivos de carácter supranacional. En la actualidad, estos fenómenos hacen ineludible planificar el territorio desde una perspectiva transnacional y no encarar las unidades espaciales de una forma insular o autista. En términos académicos estas temáticas surgen asociadas a las técnicas y métodos de la Ordenación Espacial Supranacional (OES) o Transnational Spatial Vision, un dominio científico multidisciplinar relativamente poco divulgado, pero que tendrá ciertamente un gran desarrollo en los próximos años, una vez que las estrategias para el territorio europeo, perspectivas nacionales y regionales incluidas, tienen cada vez más relación y dependencia con la implantación de iniciativas de OES.

Desde la perspectiva de la planificación, el acercamiento a los fenómenos supranacionales y a las configuraciones territoriales por ellos generados constituye una práctica cuyo origen se remonta a la década de los sesenta, aunque a lo largo de un amplio periodo de tiempo haya permanecido circunscrita a una esfera técnico-política restringida. Durante más de cuatro décadas de evolución, las experiencias de OES se han diversificado y adaptado a las realidades políticas y territoriales europeas, de modo que, actualmente, podemos identificar cuatro grandes modelos: el asociado a las principales organizaciones pan-europeas (Consejo de Europa y Unión Europea), el de base nacional, el de ámbito regional transfronterizo y, por último, el modelo de OES intermedio que comúnmente se encuadra entre la escala pan-europea y la escala nacional. En nuestra opinión este modelo intermedio ha sido el que más ha progresado en los últimos años, especialmente en términos metodológicos y científicos. Las iniciativas realizadas conforman nuevos métodos de diagnóstico y planificación en Euro- 
pa, procurando, por un lado, romper, a través de un proceso de cooperación voluntaria, los rígidos mosaicos de base nacional omnipresentes en muchos de los más preeminentes actores de la ordenación espacial, y por otro, solucionar algunos problemas espaciales impulsores de discordia y desigualdad. En este sentido, nos parece evidente que el OES de nivel intermedio, más que cualquier otro de los modelos, contribuye de manera fundamental a la percepción y comprensión del "Nuevo Espacio Europeo".

A lo largo del presente artículo procuraremos, en primer lugar, exponer brevemente algunas características básicas del OES, señalando su encuadramiento científico, sus objetivos y presupuestos genéricos de modo que podamos comprender, de alguna forma, el contexto epistemológico en que desarrollaran este tipo de iniciativas. Posteriormente, analizaremos de un modo conciso dos ejemplos de OES de nivel intermedio desarrollados en Europa, el VASAB 2010 y el VISION PLANET, con el propósito de identificar su origen y evolución, sus soportes institucionales y organizativos, sus objetivos y sus principales resultados. Por último, esbozaremos algunas ideas relativas a la implantación del OES en Europa y su evolución futura.

\section{LA ORDENACIÓN ESPACIAL SUPRANACIONAL}

\section{Propósitos y presupuestos generales}

Como ya señalamos anteriormente, las acciones relativas a la definición de estrategias espaciales transnacionales o a la ordenación espacial a escala supranacional, como ejercicio técnico y científico se enmarcan dentro de lo que actualmente se denomina Transnational Spatial Vision, Transnational Spatial Planning u Ordenación Espacial Supranacional (en castellano). La OES constituye un ámbito científico multidisciplinar que, en el sentido de identificar e implantar estrategias de ordenación espacial supranacional, conjuga, entre otros, técnicas/métodos de planificación estratégica, desarrollo regional, urbanismo, prospectiva (territorial), relaciones internacionales y geo-marketing. Su punto de vista se centra mucho más en la anulación de los efectos nefastos de las tendencias espaciales vigentes y en la coordinación de los impactos territoriales de las políticas sectoriales que en la simple ordenación física del territorio y respectiva programación del uso del suelo. Aun reconociendo que cada iniciativa de OES deberá partir de una identificación rigorosa de sus objetivos específicos, perfectamente adaptados a los agentes y territorios involucrados, podemos afirmar que de un modo general los propósitos de un ejercicio de OES se centran en los siguientes puntos.

i) Comprensión de las implicaciones transnacionales y de las tendencias de desarrollo espacial a largo plazo, de los desafíos inherentes a éstas, de los pro- 
blemas respectivos y de los diferentes conceptos de desarrollo en un contexto amplio. Actualmente, después de décadas de "planificación insular" en entidades territoriales que eran tratadas como si no tuviesen vecinos, no hay duda que el desarrollo y las perspectivas futuras de una región o país depende siempre de lo que pase fuera de su territorio, con especial relevancia para el vinculo existente con la propia evolución de los espacios adyacentes.

ii) Profundizar y mejorar el conocimiento respecto a las relaciones e interdependencias entre distintos países/territorios y las posturas de planificación y desarrollo regional/espacial de cada uno de los agentes involucrados. En este sentido es fundamental interiorizar la idea de que el interés común de un conjunto de territorios pertenecientes a diferentes países no es definido simplemente por la suma de todos los intereses individuales, pero si por el hecho de procurarse la mejor utilización de los recursos disponibles en el amplio conjunto espacial y la optimización de las ventajas inherentes a la unión de fuerzas y esfuerzos de desarrollo.

iii) Proporcionar una declaración de objetivos comunes a alcanzar en términos de estructura territorial de una unidad espacial. Estos objetivos poseen siempre, explicíta o implícitamente, una dimensión espacial y los ejercicios de OES comportan la tarea y la responsabilidad de suministrar una visión de los desafíos, riesgos y oportunidades espaciales a tener en cuenta, definiendo igualmente los instrumentos más apropiados para fomentar el correcto desarrollo.

iv) Suministrar una correcta y eficiente orientación para los procesos de ordenación espacial infranacionales y actuar para influenciar las restantes políticas sectoriales espacialmente relevantes, con la intención de maximizar los posibles efectos de sinergia.

v) Apoyar un proceso de formulación y selección de programas/proyectos de naturaleza transnacional/transfronteriza que puedan contribuir a una mejor ordenación espacial de la unidad observada. En la última década hemos asistido a un incremento de los programas e incentivos financieros específicos para los ámbitos transnacionales y transfronterizos, en especial aquellos cuya génesis es comunitaria (como por ejemplo el Progama Interreg en sus múltiples vertientes, el Programa Phare CBC, el Programa TERRA, el Programa CARDS, el Programa CREDO, etc.). En este sentido, la existencia de iniciativas de OES para los territorios incluidos en este tipo de programas constituye un instrumento fundamental, bien sea en la elaboración de los 
programas propiamente dichos, bien en la definición de criterios de selección de los proyectos a apoyar ${ }^{1}$.

Una de las características esenciales del OES que debemos destacar aquí es la enorme importancia que adquiere la componente política de la planificación y los procesos de cooperación. Estas iniciativas suponen inevitablemente concepciones y opciones políticas evidentes que, al abarcar una traducción espacial supranacional, difícilmente pueden estar exentas de un delicado proceso de cooperación con los territorios implicados. Por otro lado, los propios procesos de cooperación dependen ampliamente de la coyuntura política o geopolítica (presencia de experiencia y tradición en los procesos de cooperación, existencia de contiendas territoriales o económicas, proximidad cultural, etc.) vigente entre las unidades territoriales en cuestión, lo que per se refuerza, una vez más, la propia componente política del OES.

Como podemos deducir, el OES comporta procesos multidisciplinares complejos para los cuales no surgen metodologías y formatos institucionales universales que puedan ser directamente aplicados a todos los casos. No obstante, esto no significa que no exista un conjunto de presupuestos subyacentes a este tipo de actividades y que podemos sintetizar en los siguientes puntos (TREUNER, 2000, 14-15).

i) La realización de iniciativas de OES exige siempre un análisis válido de las perspectivas particulares de cada uno de los territorios pertenecientes a un conjunto transnacional. Este estudio deberá ser efectuado en cooperación con los respectivos vecinos utilizando categorías de descripción y análisis compatibles y consensuadas. En este sentido, una de las responsabilidades fundamentales de cuerpo técnico consiste en definir categorías de observación y previsión espacial compatibles que puedan traer un mínimo de racionalidad al proceso de definición de políticas de desarrollo espacial transnacional.

ii) Para ser entendidas y respetadas, las acciones de OES han que ser realistas y realizables. En el caso de que estas se limiten a proponer "bonitos sueños", seguramente no serán respetadas por ninguno de los agentes preeminentes y solo podrán servir, si fuera posible, de camuflaje a aproximaciones parciales no integradas, bien como para definir proyectos y programas de responsabilidad sectorial claramente limitada.

\footnotetext{
${ }^{1}$ Los primeros cuatro puntos fueran adaptados de TREUNER, 2000, pp. 11-13 y el quinto de NADIN, 2000, p. 19
} 
iii) La preparación de iniciativas de OES y de objetivos de desarrollo espacial para un conjunto amplio de territorios no exige únicamente un conocimiento suficientemente válido de las especificidades y problemas de cada uno de los territorios. Un ejercicio de OES exige igualmente que este sea complementado por una definición del interés común, que no es necesariamente la suma de todos los intereses individuales, como señalamos anteriormente.

iv) Las acciones de OES deberán incluir una parte en que se esclarezca su significado dentro de la concepción e implantación de otras políticas estructurales/sectoriales. La práctica hace notorio que las políticas sectoriales sólo incorporarán referencias de desarrollo espacial cuando (y sí) los cuerpos técnicos de planificación y ordenación espacial suministran sus interpretaciones (bien fundamentadas) acerca de los requisitos sectoriales específicos que resultan de la visión adoptada. Luego, corresponde a este universo técnico el papel de aportar sus conocimientos para volver las políticas sectoriales más eficientes, para fomentar visiones más integradas del fenómeno espacial y para identificar las soluciones adecuadas a los problemas espaciales, promoviendo, en la medida de sus posibilidades, la legitimación política y democrática del OES.

Para acabar, conviene destacar que a pesar de que el OES haya tenido un gran desarrollo en los últimos años, existe todavía una experiencia relativamente limitada en relación a los proyectos políticos, a los soportes institucionales y hasta en lo relativo a los métodos e instrumentos inherentes a la identificación e implementación de estrategias de desarrollo espacial supranacional (NADIN e SHAWT, 1998, 281), particularmente cuando los comparamos con la ordenación espacial infra-nacional. De este modo y atendiendo a la dinámica de los últimos años, es de esperar que a medio plazo este dominio de la ordenación del territorio experimente una acentuada evolución.

\section{Los principales modelos de intervención}

En Europa los orígenes del OES se encuentran en algunas iniciativas realizadas por los países del Noroeste europeo a lo largo de la década de los años sesenta (especialmente en los países del Benelux) y desde entonces se ha asistido a un significativo desarrollo y diversificación de los mismos. Un intento de clasificación de los modelos de OES existentes podrá realizarse atendiendo a su escala de intervención, de modo que en nuestra opinión el análisis de la evolución de las experiencias de OES en las últimas décadas nos permite identificar cuatro grandes modelos: $(a)$ los procesos de ámbito europeo/comunitario, $(b)$ las experiencias transnacionales de nivel intermedio, $(c)$ los ejercicios de base nacional y $(d)$ el OES aplicado a la escala regional transfronteriza. 


\section{a. Los procesos de ámbito europeo/comunitario}

En el primer caso, el origen del OES de ámbito europeo/comunitario (generalmente asociado a las "estrategias/principios de desarrollo del espacio europeo") está claramente en la actuación del Consejo de Europa (COE), o más concretamente en la Conferencia Europea de Ministros Responsables de la Ordenación de Territorio (CEMAT) cuyas reuniones se iniciaron en 1970. En esta materia, el COE, más allá de favorecer una multiplicidad de estudios ya desde finales de los sesenta, adoptó a partir de su primera reunión varias resoluciones relacionadas con las estrategias de desarrollo del espacio europeo. De entre los documentos más relevantes de la CEMAT podríamos destacar los "Fundamentos de una Política Europea de Ordenación del Territorio" de 1970, la "Carta Europea de Ordenación del Territorio" de 1983 y los "Principios Directores para el Desarrollo Territorial Sostenible del Continente Europeo" de 2000.

La otra arena primordial en el continente europeo es evidentemente la Comunidad Europea, sin embargo y hasta finales de los años ochenta las instituciones comunitarias no se interesaron de forma sustancial por este tema. En la segunda mitad de los años ochenta se comienza a cimentar la idea de que el proceso de integración europea debería ir acompañado de una adecuada coordinación de las políticas y prácticas de ordenación espacial y rápidamente se evidencia la posibilidad de concretar un conjunto de iniciativas que sirviesen de soporte a una eventual Política Europea de Ordenación Espacial y un documento base que estableciese los principios de desarrollo espacial a escala comunitaria. Este documento, que fue el resultado de un complejo y prolongado proceso de preparación y negociación, finalmente se aprobó en 1999 con el título "European Spatial Development Perspective" o "Estrategia Territorial Europea" (ETE), siendo la manifestación más tangible de la OES de ámbito comunitario.

\section{b. Las experiencias de OES de nivel intermedio}

En segundo lugar tenemos las experiencias del nivel intermedio, ejercicios de ordenación espacial transnacional pero sin abarcar grandes conjuntos de naturaleza pan-europea (involucra un número más reducido de países). Encontramos buenos ejemplos de ello en el Benelux, cuyo primer documento oficial de ordenación espacial transnacional data de $1986^{2}$, o en la región del Mar Báltico. A finales de los años noventa y claramente asociados a la Iniciativa Comunitaria Interreg IIC, surgen otros ejemplos de OES que se enmarcan dentro de este tipo, tales como el VISION PLANET, el Espacio ESTIA, el Área Metropolitana del

\footnotetext{
${ }^{2}$ Esquisse de Structure Globale du Benelux, aprobada por los ministros responsables de la ordenación del territorio de los países del Benelux en 1986 a la que se siguió la Deuxième Esquisse de Structure du Benelux, adoptada de forma definitiva en 2000.
} 
Noroeste Europeo (AMNO) y la región del Mar del Norte (NorVision). En nuestra opinión este es el modelo que en los últimos años más se ha difundido y sobre el cual existen muchos progresos metodológicos, así como algunas de las principales contribuciones en el campo de las Transnational Spatial Visions. Algunas de las virtudes que podremos encontrar en los OES de nivel intermedio están relacionadas con su ámbito territorial (verdaderamente supranacional), con la existencia de una gran multiplicidad y variedad de actores (que exige la implantación de verdaderos procesos de cooperación técnica y política, así como la concepción de nuevos sistemas organizativos supranacionales), con su enorme utilidad como puntos de referencia y orientación para la ordenación espacial a escala inferior y superior y, por último, con el carácter marcadamente voluntario y flexible reinante en su origen (por norma los actores no están sujetos a la "obligatoriedad" de participar en los procesos y estos parten muchas veces de "cero", no estando a priori sujetos a intrincadas prácticas/responsabilidades burocráticas/políticas).

\section{c. Los ejercicios de base nacional}

Las experiencias de OES se incorporaron a algunos instrumentos y procesos de planificación y ordenación espacial exclusivamente nacionales con el propósito, entre otros, de auxiliar la interpretación de las tendencias supranacionales y establecer un encuadramiento y orientación estratégica más eficaz en las políticas a implementar en el nivel infra-nacional. Algunos ejemplos de este tipo de OES se implantaron en Holanda (como por ejemplo en el Fourth report on the physical planning and perspectives in Europe de $1991 \mathrm{y}$ en el Netherlands 2030 - Discussion Document - an exploration of spatial scenarios de 1998) o en Dinamarca (dos documentos relevantes son el Denmark towards the year 2018 de 1992 y el Denmark and its neighbours: Denmark and European Spatial Planning Policy - National Planning Report for Denmark de 1997). En este campo, otros buenos ejemplos de la incorporación del OES en las estrategias de ordenación espacial nacional son el Programme directeur d 'amenagement du territoire - Project de Luxemburgo y el Aménager la France de 2020 - Mettre les territoires en mouvement francés.

\section{d. El OES aplicado a la escala regional transfronteriza}

En último lugar tenemos el OES aplicado a escala regional transfronteriza. De una forma simplificada, podemos afirmar que estas iniciativas procuran aplicar a la escala de las regiones fronterizas las lógicas y métodos inherentes a una ordenación espacial supranacional, con el fin de responder a los problemas y es- 
pecificidades concretas de este tipo de espacios. Algunos buenos ejemplos son el caso de la Metrópoli Transfronteriza de Lille, de la región de Øresund, de la frontera Alemania/Holanda (Deutch-Niederländischen Raumordungskommission), de la región Sarr-Lor-Lux-Trèves/Palatinat, de la Aglomeración Trinacional de Basilea, de la región transfronteriza Valais-Vallée d'Aoste y de la región del Rhin Superior.

Mapa 1. Algunos ejemplos de experiencias de OES de nivel intermedio en Europa

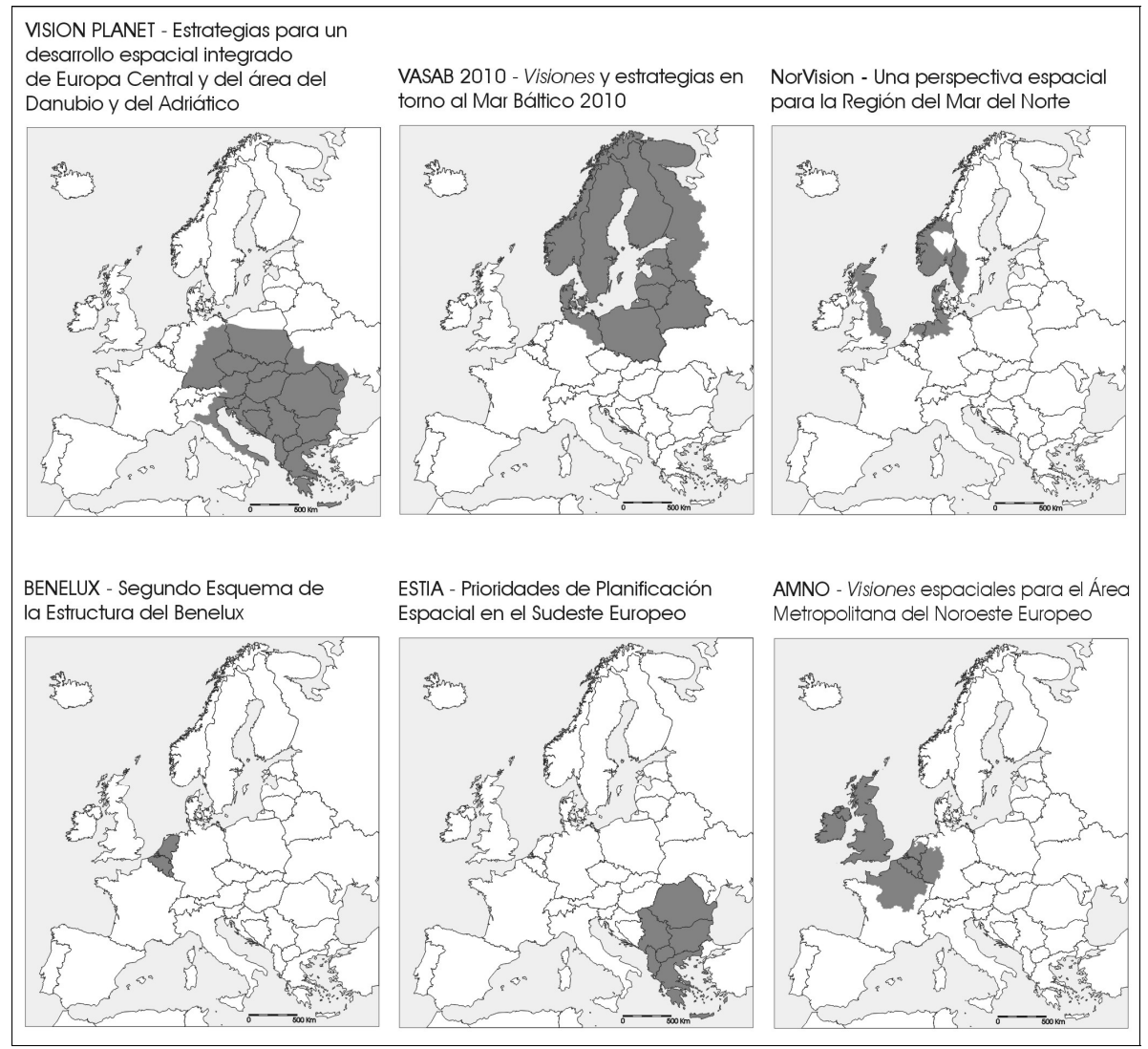

FUENTE: Elaboración propia 


\section{EJEMPLOS DE ORDENACIÓN ESPACIAL SUPRANACIONAL DE NIVEL INTERMEDIO EN EUROPA}

\section{EL VASAB 2010 (Vision and Strategies around the Baltic Sea 2010)}

La región del Mar Báltico (RMB) dio origen a uno de los proyectos más precoces de cooperación en términos de OES. Este proceso se inició en el año 1992 por iniciativa de la Conferencia de Ministros Responsables de la Ordenación del Territorio de la RMB y luego en 1994 se adoptó un informe (Towards a Framework for Spatial Development in the Baltic Sea Region. Vision and Strategies around the Baltic Sea 2010) que puede considerarse la primera Spatial Vision dentro y fuera de Europa. En 1996 nace otra contribución (From Vision to Action. Vision and Strategies around the Baltic Sea Region 2010), así como un "Plan de Acción" cuyo objetivo general consistía en implantar las estrategias anteriores. En 2001 y después de más de ocho años de experiencia en el campo del desarrollo espacial supranacional se elaboró y aprobó un nuevo documento (Vision and Strategies around the Baltic Sea 2010 Plus) comúnmente denominado VASAB 2010+ y se añadió una declaración conjunta. Ambos constituyen los elementos actualmente vigentes y demuestran el interés y dinamismo que esta materia ha tenido en la RMB.

Más allá de los documentos Vision se desarrollaron otras actividades. Por ejemplo, y hasta 1996, se realizaron algunos proyectos conjuntos relacionados con el desarrollo de grandes corredores de transportes, con el aprovechamiento turístico de actividades náuticas, con la cooperación transfronteriza y con la ordenación del territorio en regiones costeras. En esta primera fase la concreción de las actividades en el seno del VASAB contaron esencialmente con una financiación estatal bilateral, sin embargo, después de 1996 algunas acciones se desarrollaron con apoyos financieros comunitarios, concretamente de los programas Interreg IIC, Phare e TACIS. En este segundo periodo se ampliaron significativamente los campos de acción, entrando por ejemplo en el ámbito de los sistemas monitorización espacial, de los métodos y técnicas de ordenación territorial y en el desarrollo de las redes urbanas (CMSPD/BSR, 2001, 6).

Desde sus orígenes, la iniciativa VASAB ha adaptado sus objetivos al espíritu con que fue creado, pero, al mismo tiempo, no ha dejado de adaptarlos a la evolución vivida en Europa. Si, en términos genéricos, el VASAB 2010+ procura establecer una base de orientación en el campo de la ordenación espacial transnacional que pueda contribuir al desarrollo sostenible de la RMB, cuando atendemos a sus objetivos específicos, encontramos, en nuestra opinión, una postura (geo)estratégica bastante bien definida y centrada en la afirmación de la RMB en el seno de Europa. Sus objetivos son $(i)$ articular las implicaciones espaciales de la evolución económica, social y política de la RMB, (ii) incorporar los in- 
tereses y opciones de OES de la RMB en otros documentos de desarrollo espacial europeo (en especial los del COE y de la UE), (iii) establecer una puente "Este-Oeste" donde todos los países de la RMB participen en condiciones de igualdad, (iv) expresar los intereses bálticos en las futuras discusiones europeas relativas a las políticas regionales y espaciales (CMSPD/BSR, 2001,3).

Mapa 2. Región del Mar Báltico

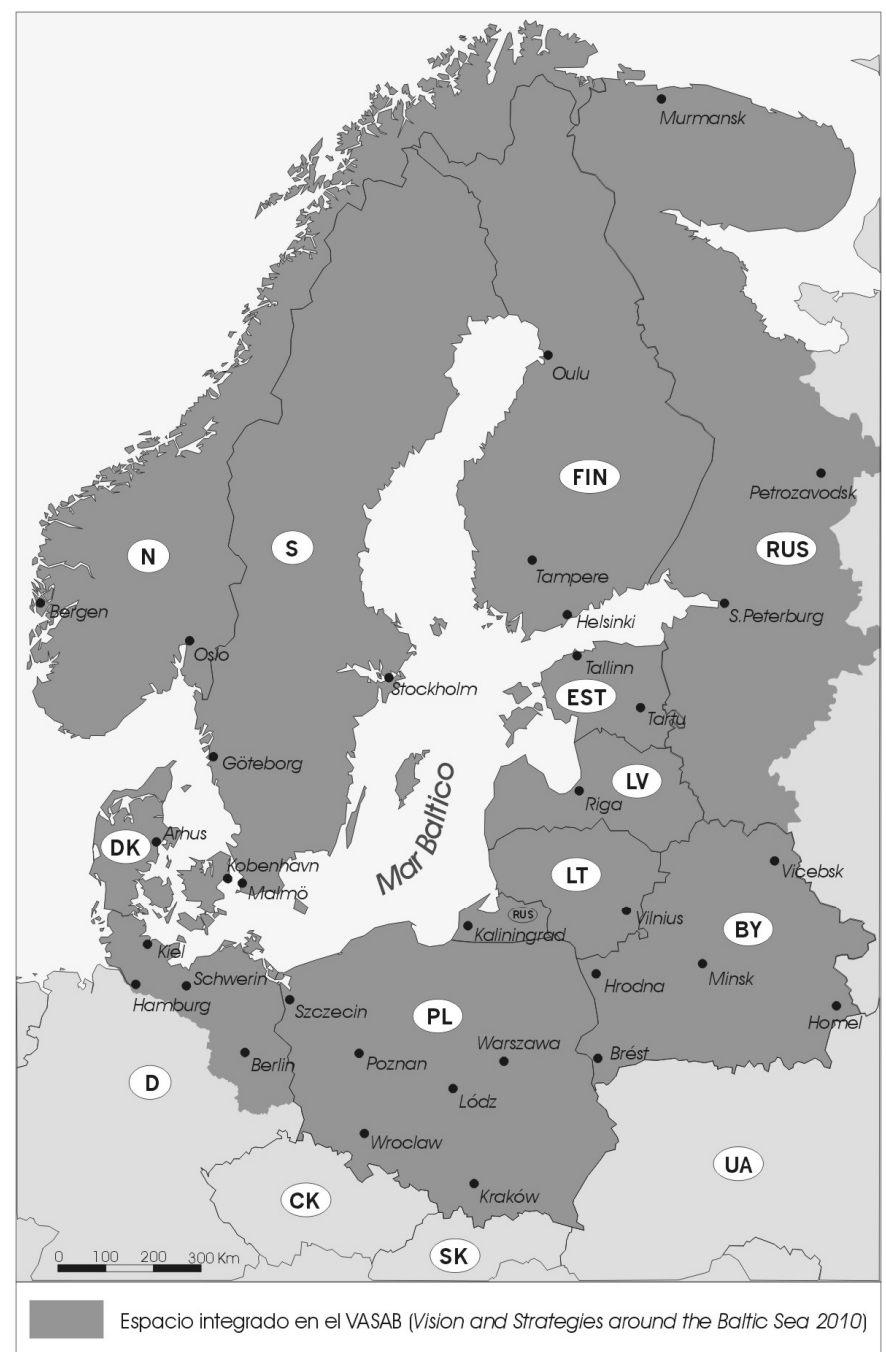

FUENTE: elaboración propia 
La iniciativa VASAB posee todos los países de la RMB, de los cuales, nueve son abarcados en su totalidad (Noruega, Suecia, Finlandia, Estonia, Letonia, Lituania, Bielorrusia, Polonia y Dinamarca) y dos parcialmente, Alemania (Estados Federados de Schleswig-Holstein, Mecklenburg-Volpommern, Brandenburg, Hamburgo y Berlín) y Rusia (regiones de Murmansk oblast, Karelia, St. Petersburg, Leningrad oblast, Pskov oblast y Kaliningrad oblast). El área comprendida es considerable, 2,33 millones de $\mathrm{km}^{2}$, aproximadamente tres cuartos del área de la UE, pero la densidad demográfica es relativamente reducida, 44 habitantes por $\mathrm{km}^{2}$. En 1998 la RMB albergaba aproximadamente 103 millones de habitantes, concentrados mayoritariamente en su sector más meridional.

Al principio la iniciativa de cooperar fue establecida por la Conferencia de Ministros, tal como referimos anteriormente, apoyada por un pequeño secretariado. El documento de 1994 fue preparado por una estructura técnica, o GFP (Group of Focal Points), constituidos por los representantes de los ministros respectivos y de algunas regiones político-administrativas. Esta estructura contaba en su totalidad con catorce elementos que trabajaban en un régimen marcadamente técnico y con el apoyo de consultores independientes. La profundización de las actividades contribuyó a que la base institucional de esta iniciativa fuese ampliada, de modo que pasó a ser constituida por el Comité de Desarrollo Espacial del Mar Báltico (CSD/BSR - Committee on Spatial Development of the Baltic Sea Region), localizado actualmente en Polonia (Gdansk) y beneficiario de un secretariado organizativo/técnico permanente y de recursos financieros propios.

En nuestra opinión el documento VASAB de 1994 presenta una clara vocación estratégica en su elaboración y presentación. En términos de objetivos y opciones políticas se identifican cuatro grandes campos, todos ellos integrantes del soporte territorial de la RMB. Estos campos son: la red urbana de importancia internacional (elemento Pearls), los vínculos efectivos y sostenidos existentes entre las ciudades (elemento Strings), las áreas con dinamismo y calidad de vida (elemento Patches) y el sistema de ordenación espacial tangible y en vigor (elemento System). Para estos elementos se definen catorce objetivos específicos, sintetizados en el CUADRO 1.

Ya con relación al documento de 2001 y en términos de estrategias y opciones políticas, el VASAB 2010+ define, partiendo del papel que debe desempeñar y de las condiciones en que se debe actuar, un conjunto de principios de intervención, de los cuales derivan seis "temas clave" sobre los que la iniciativa debe actuar en el futuro. Para cada uno de estos temas se ha determinado su contenido general, los conceptos propuestos en el ámbito del VASAB, un conjunto de proyectos potenciales, como también otras acciones relevantes para cada tema. 
Posteriormente, se presentan cinco grandes líneas de acción a las cuales cabe consumar estos "temas clave".

Cuadro 1. Dominios y designios del VASAB 2010 (1994)

I. Pearls - Red urbana de importancia internacional.

1. El sistema de ciudades disfruta de un valor acrecentado por vía de la cooperación entre la RMB y Europa.

2. El sistema de ciudades asegura una cohesión espacial.

3. Los vínculos entre las áreas urbanas y sus hinterlands rurales impulsan un equilibrio económico y ambiental.

4. Las ciudades ofrecen un ambiente atractivo para sus habitantes y para la inversión.

II. Strings - Vínculos efectivos y sostenidos existentes entre las ciudades.

5. La red de transporte de la RMB impulsa el transporte menos nocivo ambientalmente.

6. La rede de transporte provee las condiciones para una efectiva integración dentro de la RMB y de ésta con el resto del mundo.

7. La producción de energía apuesta cada vez más en las energías renovables y menos nocivas ambientalmente.

III. Patches - Las áreas con dinamismo y cualidad de vida.

8. La cooperación transfronteriza contribuye significativamente a la cohesión económica, social y espacial.

9. Las islas funcionan como el núcleo del turismo en la RMB.

10. Las zonas costeras son planeadas de forma cuidadosa y equilibrada.

11. La red de áreas naturales del Báltico está cuidada y protegida.

IV. System - Sistema de ordenación espacial tangible y en vigor.

12. La ordenación espacial contribuye para la armonización y para la cohesión espacial a través de las fronteras.

13. La ordenación espacial está basada en principios de subsidiariedad, participación y transparencia.

14. La ordenación espacial contribuye para la coordinación del planeamiento sectorial y regional.

FUENTE: CMSPD/BSR, 1994

Los "principios de intervención" referidos anteriormente derivan, en gran parte, de la cuestión de subsidiariedad y de la opción de dejar a las instituciones más adecuadas las actividades relativas al desarrollo espacial. En este contexto se considera que el VASAB $(i)$ deberá acometer sólo las tareas que pueden ser solucionadas de forma más satisfactoria a través de la cooperación transnacional, (ii) que únicamente actuará en los ambitos de cooperación transfronteriza entre entidades nacionales, regionales y locales, en el caso de que estos carezcan de soporte político a nivel nacional o nacional, (iii) que interferirá exclusivamente en procesos en los que exista una inequívoca dimensión espacial y $(i v)$ que solamente estará presente cuando los otros actores relevantes tengan interés 
en colaborar con los especialistas de desarrollo espacial (CMSPD/BSR, 2001, 17). Como señalamos anteriormente estos principios de intervención constituyen el soporte para la identificación de los seis "temas clave" para el VASAB $2010+$ que enumeraremos a continuación.

1. Cooperación de las regiones urbanas en los temas primordiales del desarrollo sostenible.

2. Desarrollo estratégico de zonas importantes para la integración transnacional de la RMB.

3. Conexión de las redes transnacionales de transporte fundamentales para la integración de la RMB en la UE.

4. Diversificación y refuerzo de las áreas rurales.

5. Desarrollo de "corredores verdes" transnacionales incluyendo el tema de los paisajes culturales.

6. Desarrollo integrado de las islas y áreas costeras.

Por último, las "líneas de acción" del VASAB 2010+ se centran en los siguientes puntos: $(i)$ recomendar medidas para otras políticas de desarrollo espacial transnacional, (ii) promover el progreso técnico y metodológico del desarrollo espacial transnacional, (iii) promover el desarrollo de proyectos de cooperación, (iv) cooperar con otras iniciativas realizadas en el seno de la RMB y (v) promover el diálogo con instituciones sectoriales.

En un esfuerzo de resumir las principales particularidades de la iniciativa VASAB, no podemos dejar de resaltar su precocidad en el ámbito del OES en Europa, así como la determinación y los medios obtenidos a lo largo de los primeros tiempos. Desde entonces, ha sido muy grande la experiencia adquirida en esta materia y los procesos e instrumentos del VASAB caminan hacia una condición más madura del OES. Este hecho es perceptible, entre otros, en el cuidado puesto en la realización de proyectos, para los cuales el VASAB sirve esencialmente como "incubadora de ideas" y como estimulador de actores. Otra característica que ha estado presente en esta evolución es la postura estratégica inherente al VASAB, sea con relación a la consolidación de la RMB como mercado regional próspero y como espacio de centralidad (europea) alternativa (al Norte), sea como lobby territorial y político en el seno de la UE. Para terminar nos queda señalar que una buena parte del éxito de esta iniciativa se debe a la existencia de un secretariado permanente y de medios financieros "propios", lo que permite que no dependa enteramente de los apoyos comunitarios, y de la opción de mantener el VASAB dentro de los objetivos y escalas de intervención 
para que fue creado, no cayendo en la tentación de sobrepasar el desarrollo espacial transnacional y el estatuto no vinculante en dirección a otros niveles de planificación y decisión.

\section{EI VISION PLANET (Strategies for Integrated Spatial Development of Central European Danubian and Adriatic Area)}

La iniciativa VISION PLANET surgió integrada dentro del Programa Interreg IIC correspondiente al espacio CADSES (Central European, Danubian, Adriatic and South-East European Space Area) y por interés de Austria y Alemania, arrancando con una primera reunión en enero de 1998. En su origen estuvo el propósito por parte de Austria de desarrollar una red de diálogo en el ámbito de desarrollo espacial ampliado a los países candidatos y los intereses por parte de Alemania en realizar un documento de OES para el Espacio CADSES, intentos que acabaran por agruparse en un único proyecto y atraer la atención de los restantes participantes. En un corto espacio de tiempo, aproximadamente dos años, fue posible poner en práctica un proceso de cooperación en el ámbito del OES y presentar realizaciones tangibles de esta iniciativa, en concreto un documento Vision y respectivos diagnósticos de soporte. En algunas de las etapas el VISION PLANET contó igualmente con apoyos financieros del Programa Phare CBC. El propósito básico de esta iniciativa consistió en desarrollar un entendimiento común de los problemas, desafíos y perspectivas de desarrollo espacial en el Espacio CADSES. Este designio se articuló en torno a dos objetivos, por un lado, formular principios, estrategias y políticas para el espacio CADSES (vector VISION) y, por otro, desarrollar un diálogo más estructurado y orientado entre los actores de ordenación del territorio existentes en el área afectada (vector PLANET).

El área abarcada por la iniciativa VISION PLANET cubre una superficie de de 1.618 millones de $\mathrm{km}^{2}$ e integra una población de aproximadamente $197 \mathrm{mi}$ llones de habitantes, que representa $23,4 \%$ de la superficie de Europa (excluyendo a Rusia) y $26,5 \%$ de su población. Está constituida por dieciocho países, trece de los cuales están integrados en su totalidad (Albania, Bosnia, Bulgaria, Croacia, República Checa, Grecia, Hungría, Macedonia, Moldavia, Rumania, República Eslovaca y Yugoslavia) y cinco solo parcialmente (Austria, Alemania, Italia, Polonia y Ucrania). Existen países que, por diversas razones, no participaron de forma efectiva en la elaboración de los principales documentos, concretamente Albania, Bosnia, Macedonia, Ucrania, Moldavia y Montenegro en cuanto parte de la República de Yugoslavia, pero en relación a los cuales es grande el interés y empeño en una participación posterior (PROJECT PANEL VISION PlANET, 2000, 1). Como podemos imaginar, atendiendo a los países considerados, las disparidades socio-económicas son enormes, una vez que este 
espacio incorpora algunas da las regiones más desarrolladas de Europa, junto con una buena parte de las más necesitadas.

Mapa 3. Espacio abarcado por la iniciativa VISION PLANET

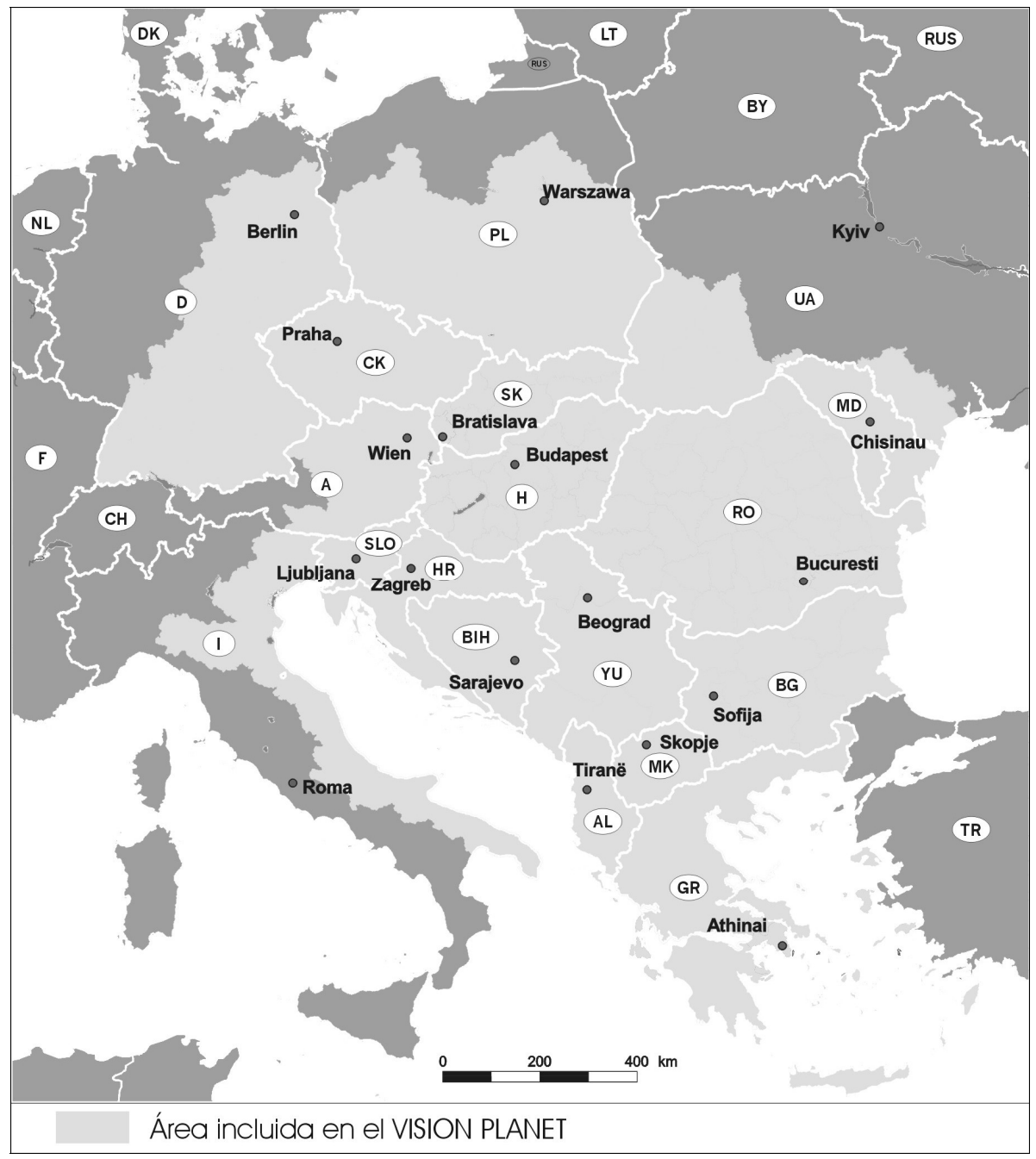

FUENTE: adaptado de PROJeCt PANEL - VISION PlANET, 2000, 6

El VISION PLANET constituye, desde su origen, un proceso desarrollado esencialmente desde un nivel técnico, con base en un régimen "bottom-up" y sin que anteriormente existiese una estructura intergubernamental de decisión. 
Los principales órganos son un Equipo de Trabajo (Working Team) que es bastante variado en su composición, comprendiendo desde universitarios y especialistas de centros de investigación hasta consultores privados, el Panel de Proyecto (Project Panel), en su mayoría ministros o representantes de los órganos de Estado responsables de la ordenación del territorio o de desarrollo regional y un Grupo Independiente de Expertos (Expert Group Menbers), todos del universo científico.

Como ya señalamos anteriormente la iniciativa se divide en dos grandes vectores, las acciones relativas al PLANET y las acciones relativas al VISION, que de algún modo son procesos paralelos. En el primer caso, los objetivos pasan por promover un entendimiento común en relación a los problemas y especificidades territoriales del Espacio CADSES y por la creación de una red de diálogo y divulgación de conocimiento entre entidades y especialistas en el campo de la planificación y ordenación del territorio (PLAnners NETwork). En el caso del VISION las acciones se centran esencialmente en la elaboración y aprobación de un documento conjunto (documento VISION) relativo a los principios, estrategias y políticas. Este documento surge en 2000 y está dividido en dos componentes, el "Background Report" (documento de cariz más técnico que sustenta las opciones y objetivos asumidos) y el documento que contiene los objetivos y las propuestas políticas de desarrollo espacial del Espacio CADSES propiamente dichas - el "Guidelines and Policy Proposals" (VISION PLANET GPP). Por medio de una Resolución este documento fue aprobado por los países y regiones involucrados el 12 de enero de 2000 en Viena, pasando a constituir el nuevo marco de referencia para las políticas de desarrollo espacial en el Área de Europa Central, del Danubio y del Adriático. El documento VISION fue elaborado por el Equipo de Trabajo, cuya coordinación estuvo a cargo del departamento federal de planificación regional alemán, contando con el apoyo del Grupo Independiente de Expertos y la supervisión del Panel de Proyecto.

En relación con los objetivos y políticas de desarrollo espacial concretos, debemos acudir al documento VISION PLANET GPP, pero y como es obvio, no podremos exponer el amplio e interesante contenido del mismo, por lo que, de forma resumida, presentaremos únicamente los elementos que consideramos más relevantes. En términos de objetivos se identifican cuatro puntos que de una forma general, surgen asociados a una buena parte de este tipo de iniciativas en Europa (PROJECT PANEL - VISION PLANET, 2000, 9):

1. Competitividad, eficiencia y crecimiento - El desarrollo espacial debe contribuir a un desarrollo económico y social más eficiente, dinámico y equilibrado a través del incremento y mejora de la competitividad de la estructura espacial, del tejido productivo y de las infraestructuras; 
2. Desarrollo equilibrado y cohesión -El desarrollo espacial debe contribuir a una estructura económica y social más equilibrada a través del incremento de la cohesión económica y social y del apoyo a las áreas con mayores problemas de progreso.

3. Protección del ambiente y del patrimonio natural - El desarrollo espacial debe contribuir a la preservación de la herencia natural y cultural a través de la promoción de un desarrollo sostenible y del uso racional de los recursos ambientales.

4. Integración - El desarrollo espacial debe contribuir a que las regiones fronterizas sean más permeables a la interacción y a los contactos, para acercar las regiones y las ciudades en términos de distancia temporal, económica y social o psicológica y para ejecutar tareas conjuntas en procesos de efectiva cooperación.

Grafico 1. Esquema organizativo de la iniciativa VISION PLANET

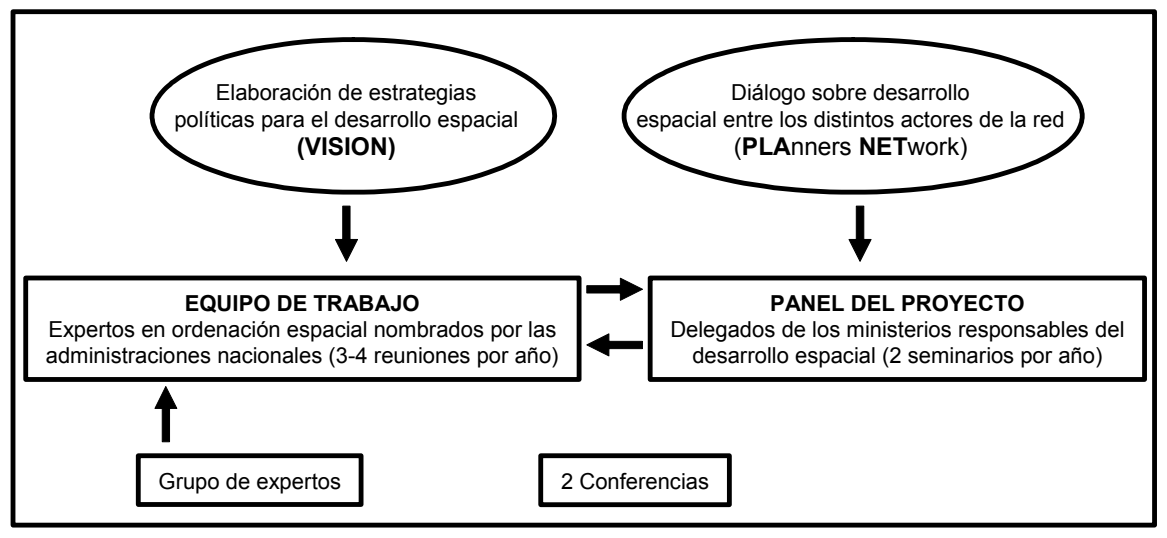

Fuente: adaptado de Project PANEL - Vision Planet, 2000, 2

En el seno del VISION PLANET estos objetivos se concretan a través de cinco campos de acción distintos (A - mejora de la estructura espacial, B - orientar el desarrollo de los asentamientos y de las ciudades, $\mathrm{C}$ - transformar las áreas rurales, D - desarrollar los transportes y las telecomunicaciones, $\mathrm{E}$ - protección del ambiente y gestión del patrimonio natural y cultural). Cada campo de actividad comporta un conjunto de tareas específicas sobre las cuales fueron finalmente presentadas (ya muy detalladas) las diversas opciones políticas. En conjunto, tenemos que reconocer que esta iniciativa presenta ya un alto grado de 
profundidad, no tanto en relación a los objetivos, pero si en relación a la minuciosidad y naturaleza desagregada y sectorial de sus opciones políticas (lo que revela la gestación eminentemente técnica del documento).

Cuadro 2. Campos de acción y tarefas específicas del VISION PLANET

A. Mejorar la estructura espacial

A.1. Combatir el incremento de las disparidades regionales

A.2. Establecer una nueva relación urbano-rural

A.3. Afrontar el problema de la periferización

A.4. Diversificación de las estructuras económicas regionales y movilización de los recursos endógenos

A.5. Implementación de un mejor acceso a la información y al conocimiento en todos los espacios

B. Orientar el desarrollo de los asentamientos y de las ciudades

B.1. Prioridades en el desarrollo del sistema urbano

B.2. Transformación de la estructura de las ciudades

B.3. Vivienda, finanzas y servicios urbanos

C. Transformar las áreas rurales

D. Desarrollar los transportes y las telecomunicaciones

D.1. Transportes

D.2. Telecomunicaciones

E. Protección del ambiente y gestión del patrimonio natural y cultural

E.1. Protección del ambiente

E.2. Gestión del patrimonio natural

E.3. Gestión de la herencia cultural

FUENTE: adaptado de PROJECT PANEL - Vision PlaneT, 2000

Por último, se definen seis áreas transnacionales de especial importancia para el desarrollo e integración del Espacio CADSES y sobre la cuales las políticas de desarrollo deberían centrar su atención. Estos grandes espacios son el "Área de Interacción de Europa Central" (espacio que articula la actual UE con los países no miembros), el área del "Pacto de Estabilidad para el Sudoeste Europeo" (espacio abarcado directa o indirectamente por los conflictos de los Balcanes), la "Región de Desarrollo de los Cárpatos" (sector más oriental que engloba las regiones más pobres y que pasará a ser frontera con la UE después de la próxima ampliación) y un conjunto de áreas fuertemente interrelacionadas con elementos naturales, la "Región del Adriático", la "Zona de Cooperación del Danubio" y el "Área de Cooperación del Mar Negro". Se considera que existen otras áreas de menor dimensión fundamentales para el OES en el Espacio CADSES, con todo se entiende que estas áreas no deberán ser definidas en el ámbito de la presente iniciativa (PROJECT PANEL - VISION PLANET, 2000, 51). 
En lo que respecta a la evolución posterior, en el momento de la aprobación del VISION PLANET GPP fueron definidas de forma relativamente concisa las expectativas futuras en relación con esta iniciativa. De entre los elementos más significativos en este ámbito podemos subrayar por ejemplo el interés en desarrollar proyectos concretos relativos a los elementos territoriales y principales tipos de áreas problemáticas identificados en el proceso de cooperación, proyectos estos que se intentarían integrar posteriormente en la Iniciativa Comunitaria Interreg III. En este campo, las estructuras del VISION PLANET podrían desempeñar funciones de apoyo y consultoría, así como podrían servir de base para la implantación del ESPON en esta área. Otro elemento relevante es la profundización de la red PLANET como instrumento para la investigación de las materias relativas al desarrollo espacial, designadamente las de interés común en el Espacio CADSES. Por último tenemos la integración del VISION PLANET en las grandes iniciativas de ordenación espacial a escala europea, nombradamente las oriundas del COE e de la UE, y su incorporación en las políticas nacionales y en otros procesos de cooperación transfronteriza o transnacional.

En un intento de síntesis, ¿cuáles son los elementos que destacaríamos en esta iniciativa? En primer lugar, el gran énfasis que se ha puesto en la reforma y transición de los países del antiguo "Bloque Socialista", con especial interés en el problema de las enormes disparidades y la necesidad de asegurar la estabilidad de estos espacios y el mantenimiento de la paz. En segundo lugar, el hecho de que el principal documento (VISION PLANET GPP) haya sido realizado con base en una aproximación de "abajo a arriba", eminentemente técnica y sin una decisión intergubernamental preliminar y que no haya sido muy complejo el proceso de su aprobación política. Aunque este posea una naturaleza informal, surgiendo simplemente como una guía de orientación no vinculante, tenemos que reconocer que fue elaborado en un periodo de tiempo bastante corto, no olvidando, sin embargo, el rigor necesario para la materia en cuestión. En términos metodológicos es de destacar el esfuerzo hecho para abandonar los diagnósticos y discusiones accesorios y la presentación por casi todos los países involucrados de sus esquemas de desarrollo espacial internos. Estos esquemas fueran tenidos en cuenta en el documento VISION, utilizando para ello una metodología común y una participación de los actores implicados. Por último, pensamos que constituye en el ámbito académico un análisis y reflexión notable sobre el Espacio CADSES y los países que lo componen, tantas veces desconocidos o ignorados desde Europa Occidental.

\section{CONCLUSIONES}

El estudio de estos dos casos nos permite tener una idea de la diversidad de acciones que se pueden realizar en el ámbito del OES en Europa y de los dos tipos de sistemas organizativos necesarios. En esto momento están en curso va- 
rias iniciativas de nivel intermedio, por ejemplo las que son financiadas por el Programa Interreg IIIB, así como innumerables proyectos de naturaleza científica relacionados con los métodos y las prácticas de ordenación espacial transnacional que, en su conjunto, seguramente establecerán aportaciones significativas a medio plazo. Podremos deducir también que el desarrollo de las iniciativas de OES depende mucho de la tradición en términos del modus operandi del planeamiento y da la voluntad/empeño político en participar. Por ejemplo y en relación al OES de nivel intermedio, en el Sudoeste Europeo este tipo de actividades son, hasta la actualidad, prácticamente inexistentes, a pesar de que estos países hayan recibido apoyos financieros que las deberían haber concretado (Interreg IIC). Existirán, como es lógico, algunas razones para este hecho, y seguramente no será porque estos países hayan permanecido inmunes a los impactos espaciales transnacionales nefastos del proceso de globalización, de la integración económica europea, de las políticas comunitarias, etc., o por encontrarse exentos de los eventuales impactos negativos del proceso de reconfiguración territorial que sufrirá la UE una vez realizada y consolidada la siguiente ampliación.

Por ultimo, el análisis de estos dos ejemplos apunta en el sentido de que los orígenes, los métodos y los soportes institucionales de las iniciativas de OES pueden variar considerablemente, pues, los propios procesos tiene que ser flexibles y adecuados a las circunstancias. El elemento vital es sin duda el interés y el empeño en cooperar, así como lo es para el proyecto europeo de una forma general, una vez que, para muchos casos, la cooperación constituye una senda imprescindible para la Paz y para una coexistencia cordial. La convivencia pacifica, la prosperidad y competitividad de Europa (y de sus regiones) necesitan de un suporte territorial igualmente competitivo, funcional y solidario. Una postura inflexible y confinada a las fronteras nacionales, aunque asegure la legítima defensa de las conveniencias de cada Estado, difícilmente contribuirá para la prosperidad y competitividad de los territorios englobados y de ningún modo patrocinará la convivencia pacífica en el seno de Europa. Una forma de asegurar que los territorios puedan disfrutar del derecho de reivindicar y proteger sus intereses, sin todavía establecer obstáculos profundos a la sana convivencia entre comunidades, es apostar por procesos de cooperación y por la búsqueda de puntos comunes y sinergias que beneficien a todos. Sea en un caso o en el otro se trata de actividades intrínsecas a la ordenación espacial supranacional, y por tanto al proceso de reorganización territorial de Europa.

\section{BIBLIOGRAFIA}

CEMAT (1970): Fundamentos de uma política europeia de ordenamento do território. CEMAT (70) 11, Estrasburgo, Conselho da Europa. 
Cemat (1983): Carta europeia do ordenamento do território. Adoptada pela Conferência Europeia dos Ministros responsaveis pelo Ordenamento do Território em Torremolinos a 20 de Maio de 1983, Estrasburgo, Conselho da Europa.

Cemat (2000): Princípios orientadores para o desenvolvimento territorial sustentável do Continente Europeu. CEMAT (2000) 7, Estrasburgo, Conselho da Europa.

CMSPD/BSR (1994): Towards a Framework for Spatial Development in the Baltic Sea Region. Vision and Strategies around the Baltic Sea 2010. Third Conference of Ministers for Spatial Planning and Development, Tallinn, 7-8 December, 1994.

CMSPD/BSR (1996): From Vision to Action. Vision and Strategies around the Baltic Sea Region 2010. Fourth Conference of Ministers for Spatial Planning and Development, Stockholm, 22 October 1996.

CMSPD/BSR (2001): Vision and Strategies around the Baltic Sea 2010 Plus. Wismar Declaration and Vasab 2010 Plus - Spatial Development Action Programme. Fifth Conference of Ministers for Spatial Planning and Developmnent, Wismar, 20-21 September, 2001.

CoMissão EuROPEIA (1999): EDEC - Esquema de Desenvolvimento do Espaço Comunitário, Luxemburgo, Serviço das Publicações Oficiais das Comunidades Europeias.

CSD/BSR (1998): Spatial planning for sustainable development in the Baltic Sea Region. A VASAB 2010 contribution to BALIC 21. Gdansk, Committee on Spatial Development of the Baltic Sea Region, April 1998.

CSD/BSR (2001): VASAB 2010 Plus. Spatial Development Action Programme - Backgrond documents, Gdansk, Committee on Spatial Development of the Baltic Sea Region - PLANCO Consulting, February 2001.

DATAR (2000): Aménager la France de 2020 - Mettre les territoires en mouvement. Paris, DATAR.

MAT (1999): Programme directeur d'amenagement du territoire - Project, Ministere de l'Amenagement du Territoire, Grand-Duche de Luxembourg, Mai 1999.

Mev (1997): Denmark and its neighbours: Denmark and European Spatial Planning Policy - National Planning Report for Denmark. Copenhagen, Ministry of Environment and Energy, Spatial Planning Department. 
Ministry OF THE ENVIRONMENT (1992): Denmark towards the year 2018, Copenhagen, Ministry of the Environment.

NADIN, V. y SHAW, D. (1998): «Transnational spatial planning in Europe: the role of Interreg IIC in the UK», Regional Studies, vol. 32, n. ${ }^{\circ}$, pp. 281-299.

NADIN, Vincent (2000): «Review of existing transnational spatial planning documents», en (SCHINDEGGER, F.) Reviewing transnational spatial planning, Vienna, Austrian Institute for Regional Studies and Spatial Planning (ÖIR), pp. 17-27.

PROJECT PANEL - VISION PlANET (2000): Strategies for integrated spatial development of the Central European Danubian and Adriatic Area-Guidelines and Policy Proposals, Approved at the seminar of the Project Panel on the 12 January in Vienna, 2000.

RIJKSPLANOLOGISHE DIENST (1991): Fourth report on the physical planning and perspectives in Europe, The Hague, Rijksplanologishe Dienst.

TREUNER, Peter (2000): «Functions and features of transnational spatial visions», en (SCHINDEGGER, F.) Reviewing transnational spatial planning, Vienna, Austrian Institute for Regional Studies and Spatial Planning (ÖIR), pp. 11-15.

VROM (1998): Netherlands 2030 - Discussion Document - an exploration of spatial scenarios. The Hague, Ministry of Housing, Physical Planning and the Environment.

VROM (2001) - Ruimte maken, ruimte delen. Vijfde nota over ruimtelijke ordening 2000/2020. The Hague, Ministerie van Volkshuisvesting, Ruimtelijke Ordening en Milieubeheer.

WORKING TEAM - VISION PLANET (2000): Strategies for integrated spatial development of the Central European Danubian and Adriatic Area - Background report. Working Team from VISION PLANET, April 2000.Co

RESUMEN: En Europa resulta cada vez más trascendente no abordar las distintas unidades espaciales desde una perspectiva “insular", integrándolas convenientemente en su marco transnacional. En este contexto, han ganado especial relevancia las técnicas y métodos de ordenación espacial supranacional, cuyo origen se remonta a los años sesenta. Existen varios modelos de este tipo de iniciativas, entre los cuales destacamos los de nivel intermedio, encuadrados entre la escala pan-europea y la escala nacional. Un aná- 
lisis más pormenorizado de algunas de estas iniciativas, caso del VASAB 2010 y del VISION PLANET, evidencia el carácter estratégico de sus objetivos y la gran flexibilidad de métodos y soportes institucionales. El estudio de estas experiencias pone de manifiesto igualmente la importancia de la ordenación espacial supranacional tanto con relación a la competitividad y sostenibilidad del territorio europeo, como a la estabilidad y coexistencia pacífica en Europa.

PALABRAS CLAVE: Ordenación espacial supranacional, Europa.

ABSTRACT: In Europe it is more and more transcendent not to approach the different space units from an insular perspective, integrating them meetly in their transnational mark. In this context, they have won special relevance the techniques and methods of planification space supranational whose origin sixty go back a years. Several models of this type of initiatives exist, among which highlight those of intermediate level, framed between the bread-European scale and the national scale. A more itemized analysis of some of these initiatives, case of the "Vasab 2010" and of the "Vision Planet", evidences the strategic character of its objectives and the great flexibility of methods and institutional supports. The study of these experiences shows equally the importance of the planification space so much supranacional with relationship to the competitiveness and sustentability of the European territory, like to the stability and peaceful coexistence in Europe.

KEY WORDS: Transnational spatial planning, Europe.

$\boldsymbol{R} \boldsymbol{E} \boldsymbol{S U} \boldsymbol{M} \boldsymbol{E}:$ En Europe, il devient de plus en plus essentiel de ne pas traiter les diverses unités territoriales sous une optique « insulaire », mais de les intégrer convenablement dans leur cadre transnational. Dans ce contexte, l'importance des techniques et des méthodes d'aménagement supranational de l'espace, nées dans les années soixante, s'est accrue. Il existe plusieurs modèles de ce type d'initiative, parmi lesquels on fait une mention spéciale des ceux appartenant au niveau intermédiaire, entre les niveaux paneuropéen et national. Une analyse plus en détail des quelques-unes des ces initiatives, telles que le VASAB 2010 et le VISION PLANET, met en évidence le caractère stratégique de leurs objectifs ainsi que la grande souplesse des méthodes et des supports institutionnels. En outre, l'étude de ces expériences souligne également l'importance de l'aménagement supranational de l'espace, aussi bien pour la compétitivité et la durabilité du territoire européen que pour la stabilité et la cohabitation pacifique au sein de l'Europe.

MOTS-CLÉS: Aménagement transnational de l'espace, Europe.

RESUMO: Na Europa resulta cada vez mais transcendente não abordar as distintas unidades espaciais desde uma perspectiva "insular", integrando-as de maneira 
conveniente no seu marco transnacional. Neste contexto, têem ganho especial relevo as técnicas e métodos de ordenação espacial supranacional, cuja origem remonta aos anos sessentas. Existem vários modelos deste tipo de iniciativas, entre os quais destacamos aqueles do nível intermédio, enquadrados entre a escala pan-europeia e a escala nacional. Uma análise mais pormenorizada dalgumas destas iniciativas, caso do VASAB 2010 e do VISION PLANET, torna evidente o carácter estratégico dos seus objectivos e a grande flexibilidade de métodos e suportes institucionais. O estudo destas experiências põe de manifesto igualmente a importância da ordenação espacial supranacional tanto com relação à competitividade e à sustentabilidade do território europeu, quanto à estabilidade e à coexistência pacífica na Europa.

PALAVRAS CHAVE: Ordenação espacial supranacional, Europa. 